\title{
Mineralocorticoid receptor splice variants in different human tissues
}

\author{
Lucia Wickert, Matthias Watzka, Ursula Bolkenius, Frank Bidlingmaier and Michael Ludwig \\ Department of Clinical Biochemistry, University of Bonn, Sigmund Freud Strasse 25, 53105 Bonn, Germany \\ (Correspondence should be addressed to L Wickert)
}

\begin{abstract}
The mineralocorticoid receptor (MR), a member of the steroid receptor family, acts as a transcription factor and mediates both aldosterone and cortisol effects. Aldosterone specificity in some tissues results from the inactivation of competing cortisol into cortisone by $11 \beta$-hydroxysteroid dehydrogenase. In other tissues MR and the glucocorticoid receptor show overlapping physiological effects or may act together by forming a heterodimer. An additional MR splice variant $(M R+4)$ has been found in different mRNA samples from rat tissues and human white blood cells, thereby implying additional modes of MR-regulated effects. We therefore looked for the presence of these two MR-mRNA isoforms in human classical aldosterone target tissues and various other tissues. MR-mRNA was found in all samples investigated, thereby showing the expression of MR to be more abundant than has been observed thus far. In addition, the MR+4-mRNA variant was also found in all the tissues examined.
\end{abstract}

European Journal of Endocrinology 138 702-704

\section{Introduction}

The human mineralocorticoid receptor (MR) mediates aldosterone action in different target tissues, e.g. kidney, colon, and salivary and sweat glands. The hormonereceptor complex acts as a ligand-dependent transcription factor by controlling the expression of several genes, thereby regulating ion balance. The $60-90 \mathrm{~kb}$ long MR gene is composed of ten exons including two untranslated exons (1a, 1b) which are controlled by separate promoters. They are alternatively spliced to two $6 \mathrm{~kb}$ long mRNA isoforms that differ in their $5^{\prime}$ untranslated region and are expressed in a tissue specific manner (1). Both mRNA subtypes result in the same $107 \mathrm{kDa}$ receptor protein $(2-4)$. In addition, different splice site usage (donor splice site: end of exon 3 or intron c) of the MR-mRNA has been observed in various rat tissues and human white blood cells. This leads to an insertion of $12 \mathrm{bp}$ and to a transcript of 988 $(\mathrm{MR}+4)$ amino acids (5).

As a characteristic feature MR and other members of the steroid receptor subfamily (receptors for glucocorticoids, progesterone, androgen and estrogen (6)) contain two zinc fingers which, in case of the wild-type MR, are separated by $45 \mathrm{bp}$. In the $\mathrm{MR}+4$ variant the additional four amino acid residues enlarge this zinc finger spacing region, suggesting different modes of receptor-mediated gene regulation by these two MR proteins. For this reason we focused our attention on the presence of the two MR splice variants in ten different human tissues, and established their ratio by mRNA quantification.

\section{Materials and methods}

Normal human tissues were obtained at surgery from the University Hospital, Bonn and the Johannes Hospital, Bonn after consent had been obtained in accordance with local institutional guidelines. Samples were either immediately frozen (within $5 \mathrm{~min}$ ) or microdissected (glandular breast tissue) and stored at $-80^{\circ} \mathrm{C}$. RNA was extracted by the use of the Trizol reagent (Gibco-BRL, Paisley, Strathclyde, UK). Reverse transcription (RT) was performed with Expand Reverse Transcriptase (Boehringer Mannheim, Mannheim, Germany) according to the instructions of the manufacturer. The cDNA was amplified with the following primers: P1F (5'CGGCGACCTGTCGTCTAGAAG3'), P2Fn (5'CCAGAAAATGTATCAAGCTCT-AC3'), P3R (5'CC AACTTCTTTGACTTT-CGTG3'), P4Rn (5'CTTCTTTG ACTTTCG-TGCTCC3'). For mRNA quantification a nested, fluorescence-labeled forward primer (P2Fn) was used in the second PCR and the resulting PCR products were separated on a $6 \%$ denaturating polyacrylamide gel. Peak areas corresponding to the two MR splice variants were detected on a 373A automatic sequencer (Applied Biosystems, Foster City, CA, USA) and analyzed with the Genescan program (Applied 


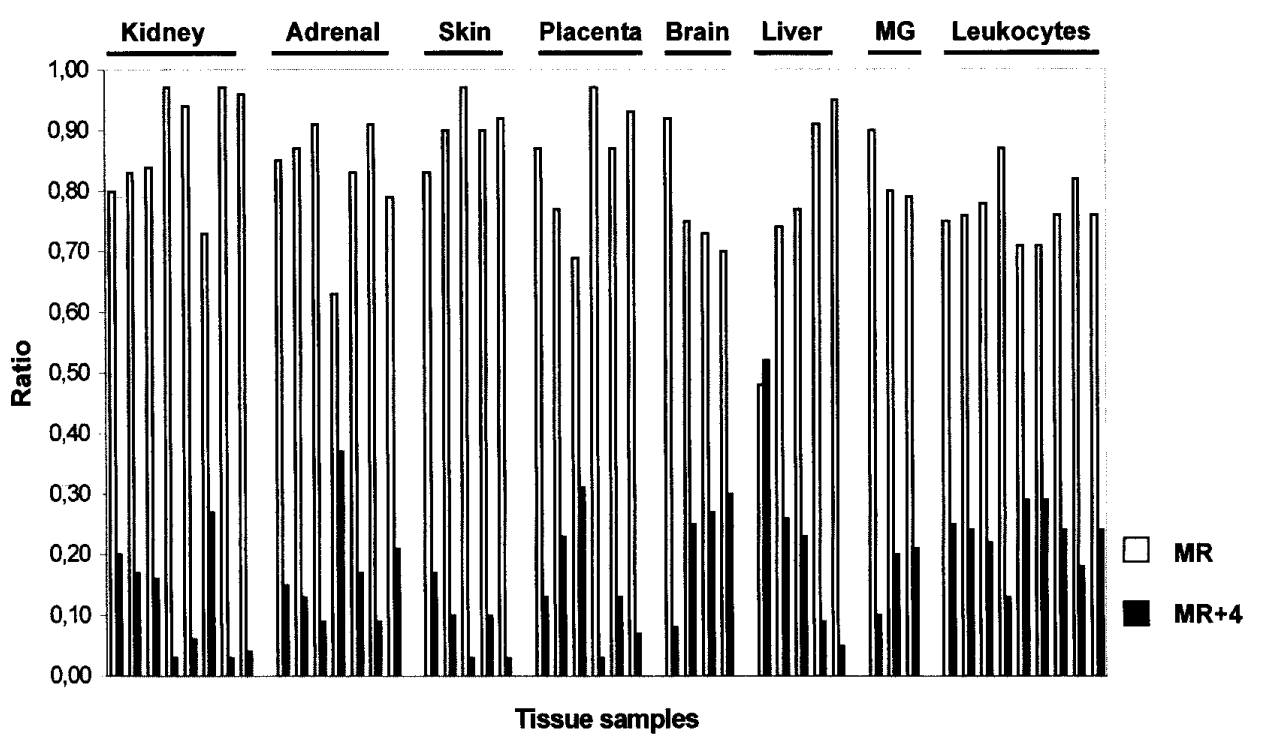

Figure 1 Ratio of MR-mRNA splice variants in different human tissues. MG=mammary gland.

Biosystems, Version 1.2.1). All samples were examined twice. As a sequence control, an aliquot of the purified PCR product served as a template in a PRISM Ready Reaction Dye Terminator Cycle Sequencing procedure (Applied Biosystems) with AmpliTaq FS (Applied Biosystems) using the $373 \mathrm{~A}$ sequencer.

\section{Results}

In contrast to Northern blotting, the use of nested fluorescence-labeled primers and a semiautomated sequencer allows the detection of mRNA species even at low levels (7). Since the sequence between the two MR-mRNA splice variants differs by only 12 bp (fragment size 290/302 bp), identical amplification kinetics during PCR can be expected, in the absence of any preference for the smaller template. We coamplified both splice variants, and PCR product analysis revealed the presence of the two MR isoforms in all human tissues tested. Ratios obtained from the individual tissue samples are shown in Fig. 1.

As can be seen, not only classical aldosterone target tissues (kidney, skin), but also adrenal and mammary gland, as well as placenta, showed expression of the MR gene and an approximate splice variant ratio of 0.85:0.15 (MR-mRNA:MR+4-mRNA). When compared with aldosterone target tissues samples from brain, those from liver and leukocytes revealed a tendency to a very slight increase in the MR+4-mRNA content. However, different ratios were obtained in various samples of each tissue investigated (e.g. the content of the wild-type MR-mRNA in nine leukocyte samples ranged between 0.71 and 0.87 ). Interestingly, samples obtained from liver tissue gave nonhomogeneous results. One sample showed a nearly equal ratio (0.48:0.52) of the two MR-mRNA isoforms, whereas two samples showed ratios of 0.7:0.3 and 0.9:0.1 respectively. In the cases of kidney and skin, the amount of the wild-type MR-mRNA was more than $97 \%$, so MR+4-mRNA content was close to the detection limit. It remains to be elucidated whether these tissues actually express the low levels of $\mathrm{MR}+4$ transcript. In addition, we have found both MR-mRNA splice variants in a pool of three samples of prostate (ratio 0.79:0.21) and two samples of ovarian tissue (ratio 0.87:0.13) (data not shown).

\section{Discussion}

RT-PCR technology combined with nested PCR enabled us to detect even low levels of MR-mRNA transcripts in tissues so far not known to express the receptor (e.g. placenta). These are the first data on the expression of MR-mRNA, in parallel with the presence of placental $11 \beta$-hydroxysteroid dehydrogenase type 2 observed by Petrelli et al. (8). In addition we have demonstrated in a variety of human tissues the splice variant previously shown to be present in rat tissues and in human white blood cells (5).

All tissues tested showed the MR+4-mRNA at lower abundance than wild-type MR-mRNA, and in one sample from kidney and skin the MR+4 RT-PCR product was close to the detection limit. The finding of almost equal amounts of both splice variants in one sample of human liver is supported by comparable findings obtained from rat liver (5). Investigations of the relative stability of rat brain MR and glucocorticoid receptor (GR) mRNA showed positive signals corresponding to both receptors $24 \mathrm{~h}$ postmortem (9). The liver data may therefore represent a particular physiological state for the tissue sample rather than an artefact of mRNA degradation over the time of sample collection and 
freezing. It is also possible that the data, despite being obtained from healthy tissue samples, might reflect altered cell metabolism in response to a particular disease state. We detected both splice variants in placenta, prostate and ovarian tissue. So far it is unknown if the variant mRNA is translated into receptor protein and, if so, what function MR might have in these tissues.

Alternative splicing is not confined to the MR, but has been found to be present in other corticosteroid receptors. For example, compared with the classical GR found in Old World primates and man (10), the GR of the New World primate marmoset also contains an additional amino acid (arginine; GR +1 ) in the interfinger region. In the rainbow trout (Onchorhynchus mykiss) another GR splice variant with nine amino acids added $(\mathrm{GR}+9)$ has been found exclusively in gill, intestine, skeletal muscle, kidney and liver (11); mRNA obtained from fish testis contains two GR splice variants, GR+9 and GR, lacking this insert (12). The observed MR and GR mRNA isoforms in all cases arise from the alternative use of a donor splice site between exon 3 and 4, and the resultant difference at the protein level may represent another pathway of steroid receptor action.

Further experiments to address these questions might also explore differences in sex- and/or age-dependent ratios of the two MR isoforms.

\section{Acknowledgements}

We are indebted to Drs A Hirner, P Albers and M Ansari for providing us with tissue samples. This work was supported by a grant from the Deutsche Forschungsgemeinschaft (Bi306/3-2).

\section{References}

1 Zennaro MC, Farman N, Bonvalet JP \& Lombès M. Tissue-specific expression of $\alpha$ and $\beta$ messenger ribonucleic acid isoforms of the human mineralocorticoid receptor in normal and pathological states. Journal of Clinical Endocrinology and Metabolism 199782 1345-1352.

2 Arriza JL, Weinberger C, Cerreli G, Glaser TM, Handelin BL, Housman DE \& Evans RM. Cloning of human mineralocorticoid receptor complementary DNA: structural and functional kinship with the glucocorticoid receptor. Science $1987237268-275$.

3 Zennaro MC, Keightley MC, Kotelevtsev Y, Conway GS, Soubrier F \& Fuller PJ. Human mineralocorticoid receptor genomic structure and identification of the expressed isoforms. Journal of Biological Chemistry $199527021016-21020$.

4 Litwak SJ, Gold PW \& Whitfield Jr HJ. The human mineralocorticoid receptor gene promoter: its structure and expression. Journal of Steroid Biochemistry and Molecular Biology 199658 495-506.

5 Bloem LJ, Guo C \& Pratt JH. Identification of a splice variant of the rat and human mineralocorticoid receptor genes. Journal of Steroid Biochemistry and Molecular Biology 199555 159-162.

6 Evans RM. The steroid and thyroid hormone receptor superfamily. Science $1988240889-895$.

7 Watzka M, Waha A, Koch A, Schmutzler RK, Bidlingmaier F, von Deimling A, Klingmüller D \& Stoffel-Wagner B. An optimized protocol for mRNA quantification using nested competitive RTPCR. Biochemical and Biophysical Research Communications 1997 $231813-817$.

8 Petrelli MD, Lim-Tio SS, Condon J, Hewison M \& Stewart PM. Differential expression of nuclear $11 \beta$-hydroxysteroid dehydrogenase type 2 in mineralocorticoid receptor positive and negative tissue. Endocrinology 1997138 3077-3080.

9 Wetzel DM, Bohn MC \& Hamill RW. Postmortem stability of mRNA for glucocorticoid and mineralocorticoid receptor in rodent brain. Brain Research 1994649 117-121.

10 Brandon DD, Markwick AJ, Flores M, Dixon K, Albertson BD \& Loriaux DL. Genetic variation of the glucocorticoid receptor from a steroid-resistant primate. Journal of Molecular Endocrinology 1991 7 89-96.

11 Ducouret B, Tujague M, Ashraf J, Mouchel N, Servel N, Valotaire Y \& Thompson EB. Cloning of a teleost fish glucocorticoid receptor shows that it contains a deoxyribonucleic acid-binding domain different from that of mammals. Endocrinology $19951363774-$ 3783.

12 Takeo J, Hata J, Segawa C, Toyohara H \& Yamashita S. Fish glucocorticoid receptor with splicing variants in the DNA binding domain. FEBS Letters 1996389 244-248.

Received 22 December 1997

Accepted 9 February 1998 